\title{
Single choroideremia Gene in Nonmammalian Vertebrates Explains Early Embryonic Lethality of the Zebrafish Model of Choroideremia
}

\author{
Mariya Moosajee, ${ }^{1}$ Merrin Tulloch, ${ }^{1,2}$ Rudi A. Baron, ${ }^{2}$ Cheryl Y. Gregory-Evans, ${ }^{1}$ \\ José B. Pereira-Leal, ${ }^{3}$ and Miguel C. Seabra ${ }^{2,3,4}$
}

\begin{abstract}
Purpose. Mutations of the $C H M$ gene underlie the X-linked chorioretinal degeneration choroideremia (CHM). The affected gene product, Rab Escort Protein (REP)1, mediates the posttranslational prenyl modification of Rab GTPases. In patients with CHM, the related REP2 partially compensates for the loss of function of REP1. The objective of this investigation was to study the natural history of disease in a zebrafish model of CHM.
\end{abstract}

Methods. Zebrafish $\mathrm{chm}^{-/-}$were bred and subjected to extensive histologic analysis and TUNEL assays, and cellular extracts were used for immunoblot and in vitro prenylation assays. A detailed evolutionary analysis was performed on the REP family.

RESults. The retina of $\mathrm{chm}^{-/-}$zebrafish develops normally for the first 4 days postfertilization (dpf) but that catastrophic multilayer degeneration synchronous with severe multisystem disease follows. Mean survival time is $4.8 \mathrm{dpf}$. At the onset of generalized disease, a significant reduction in rep expression levels and activity, with unprenylated rabs accumulating in the cytosol was demonstrated. Extensive bioinformatic analysis of the REP family of proteins revealed a single rep isoform in fish and other nonmammalian vertebrates and invertebrates that is similar to mammalian REP1.

Conclusions. REP1 appears to be the ancestral gene in the family, whereas the intronless REP 2 gene is restricted to the mammalian lineage. The results of this study propose that in $\mathrm{chm}^{-/-}$zebrafish, maternally derived rep allows initial successful development of the embryo, but its gradual loss leads to multisystem disease and invariably to lethality. In its current form, the $\mathrm{chm}^{-1-}$ zebrafish has limited usefulness. (Invest Ophthalmol Vis Sci. 2009;50:3009-3016) DOI:10.1167/iovs.082755

From ${ }^{1}$ Clinical Neuroscience, Division of Neuroscience and Mental Health, and ${ }^{2}$ Molecular Medicine, National Heart and Lung Institute, Imperial College London, London, United Kingdom; ${ }^{3}$ Instituto Gulbenkian de Ciência, Oeiras, Portugal; and ${ }^{4}$ Faculdade de Ciências Médicas, Universidade Nova de Lisboa, Lisboa, Portugal.

Supported by the Wellcome Trust; Foundation Fighting Blindness USA; Choroideremia Research Foundation; Fight for Sight; the Kennerley-Banks Fund, St. Mary's Hospital Trustees, London, United Kingdom (MM); and an MRC studentship (MT)

Submitted for publication August 20, 2008; revised October 17 and December 4, 2008; accepted April 15, 2009.

Disclosure: M. Moosajee, None; M. Tulloch, None; R.A. Baron, None; C.Y. Gregory-Evans, None; J.B. Pereira-Leal, None; M.C. Seabra, None

The publication costs of this article were defrayed in part by page charge payment. This article must therefore be marked "advertisement" in accordance with 18 U.S.C. $\$ 1734$ solely to indicate this fact.

Corresponding author: Miguel C. Seabra, Molecular Medicine, National Heart and Lung Institute, Imperial College London, London, SW7 2AZ, UK; m.seabra@imperial.ac.uk.
$\mathrm{C}$ horoideremia (CHM; Online Mendelian Inheritance in Man [OMIM] 313100) is a progressive chorioretinal degeneration with X-linked inheritance. ${ }^{1,2}$ Its estimated incidence is 1 in $50,000,{ }^{2}$ and this slow-onset disease causes night blindness in affected teenage males that progresses to loss of peripheral vision and complete blindness two to three decades after onset. CHM is characterized by slow progressive loss of the RPE and choroid in scalloped areas and atrophy of the photoreceptor cell layer. Loss of the choriocapillaris and preservation of the large choroidal vessels result in a characteristic fluorescein angiogram pattern. Heterozygous female carriers do not usually develop blindness but do have patchy areas of chorioretinal atrophy. ${ }^{1,2}$

The CHM gene was identified as encoding Rab Escort Protein (REP1), ${ }^{3-5}$ with disease caused by loss-of-function mutations leading to a truncated, nonfunctional, or rapidly degraded protein. ${ }^{1}$ A homologous protein, REP2, is the product of an intronless gene on chromosome $1 .^{6}$ REPs are necessary for successful prenyl (geranylgeranyl [GG]) modification of the Rab family of small GTPases. REPs bind newly synthesized Rabs and present them to Rab GG transferase (RGGT), which catalyzes the transfer of prenyl groups to the Rabs. ${ }^{7}$ REP then binds the prenylated Rab and delivers it to its target membrane, to which the Rab is anchored by its prenyl group. ${ }^{8}$ Rab proteins regulate membrane transport pathways, including vesicle formation, organelle movement, and membrane tethering/fusion, by residing in the membrane and interacting with a range of effector molecules in target vesicle membranes.9 Other small GTPases are prenyl lipid modified by GG transferase type I and farnesyl transferase. ${ }^{10}$

Rat tissue studies have shown ubiquitous expression of both REPs, and the absence of REP1 in CHM may be compensated for by REP2 in most tissues. ${ }^{11,12}$ However, a subset of Rabs, such as Rab27a in CHM lymphoblasts, remains selectively underprenylated in the absence of REP1. ${ }^{13}$ The recently created CHM mouse model carries a tissue-specific, tamoxifeninducible conditional knockout of the Rep1 gene. ${ }^{14}$ Heterozygous females show early onset and progressive retinal degeneration similar to human CHM. Generation of tissuespecific knockouts of REP1 in the RPE and photoreceptors suggested cell-autonomous degeneration associated with different subsets of underprenylated Rabs in each cell type. Previous attempts to develop a REP knockout mouse were unsuccessful because of embryonic lethality in males and heterozygous females when the gene is inherited from a heterozygous mother. ${ }^{15}$ This was caused by defects in trophoblast vascularization and development. ${ }^{16}$

Zebrafish have several advantages over the mouse for use as a model for the vertebrate retina. ${ }^{17}$ Zebrafish undergo extremely rapid extrauterine embryogenesis, throughout which they remain transparent. Their retinal architecture is similar to that of humans, with a rich cone density and retinal lamination reaching an adultlike state by 72 hours postfertilization (hpf). Additionally, zebrafish have a 3-month generation time; each 
breeding pair can produce up to 200 eggs per week, and animal husbandry costs are significantly lower than for mice. A zebrafish strain with a loss-of-function mutation in the orthologous CHM gene was recently described, created by random ENU ( $N$-ethyl- $N$-nitrosourea) mutagenesis and identified in a screen for balance and hearing defects. ${ }^{18}$ Positional cloning revealed a nonsense mutation $(\mathrm{C} \rightarrow \mathrm{T})$ in the second exon of rep1, a gene homologous to REP1, at residue 32 of a 666-amino acid protein. ${ }^{18}$ Mutant fish exhibited a variety of phenotypes culminating in death by 6 days postfertilization (dpf). ${ }^{18}$ The retinal phenotype was subsequently analyzed by Krock et al. ${ }^{19}$ in more detail at one developmental time point, $4.5 \mathrm{dpf}$. At this stage, the mutant fish exhibited degeneration of the RPE and photoreceptors, with complete loss of visual function as measured by electroretinography. ${ }^{19}$ With the use of transmission electron microscopy, photoreceptor layers were found to be disheveled and degenerating, and melanosomes in the RPE cells were smaller and immature and had reduced density compared with those in the wild-type retina. In addition, photoreceptor outer segment material was not effectively eliminated from the mutant RPE, resulting in the development of large vacuoles in this layer. ${ }^{19}$ However, the natural history of the disease has not been addressed in previous studies. To understand the processes underlying CHM in the zebrafish and to assess its usefulness as a model for human disease, we sought to further characterize the pattern of retinal degeneration and to correlate this with human pathology. Here we report catastrophic degeneration of the retina after $4.5 \mathrm{dpf}$. We suggest that the retinal and extraretinal phenotypes are caused by a lack of compensatory REP2, which evolved uniquely in the mammalian lineage. Absence of full-length REP is a lethal mutation in zebrafish; however, some maternal REP protein persists until approximately 4 to $5 \mathrm{dpf}$, allowing successful prenylation of rab proteins in the developing $\mathrm{chm}$ embryos. Our data suggest that once the maternal supply is exhausted, generalized Rab GTPase dysfunction may trigger multisystem failure and death.

\section{Materials ANd Methods}

\section{Aquaculture}

Zebrafish (wild-type $\mathrm{AB}$ and $c h m^{\text {rus48 }}$ ) were bred and maintained in the Imperial College animal facility according to standard protocols and the guidelines of the ARVO Statement for the Use of Animals in Ophthalmic and Vision Research. ${ }^{20}$ Embryos were raised at $28.5^{\circ} \mathrm{C}$ in facility water. The developmental stages are given in hpf or dpf, in accordance with morphologic staging criteria. ${ }^{21}$

\section{Histologic and Morphologic Studies}

Zebrafish embryos were fixed in $4 \%$ paraformaldehyde/PBS overnight at $4^{\circ} \mathrm{C}$. Samples were serially dehydrated in alcohol (5-minute washes in $50 \%, 70 \%, 90 \%, 3 \times 100 \%$ ) before they were embedded in paraffin wax, and 5- $\mu \mathrm{m}$ microtome sections were stained with hematoxylin and eosin. Sections were imaged with a camera (SIS ColorView IIIu; Olympus, Tokyo, Japan) mounted on a microscope (CKX41; Olympus). Wholemount zebrafish were imaged with a camera (DFC300 FX; Leica, Wetzlar, Germany) mounted on a fluorescence stereomicroscope (MZ16F; Leica).

\section{TUNEL Assay}

Wild-type and chm embryos were treated with $0.2 \mathrm{mM}$ phenylthiourea (Sigma, St. Louis, MO) at $10 \mathrm{hpf}$ to prevent pigment formation and were incubated at $28.5^{\circ} \mathrm{C}$ until $5 \mathrm{dpf}$. After fixation in $4 \%$ paraformaldehyde overnight at $4^{\circ} \mathrm{C}$, embryos were washed in PBS three times. Retinal sections were prepared as outlined and were dewaxed by two washes in clearing agent (Histoclear; National Diagnostics, Atlanta, GA) followed by two washes in $100 \%$ ethanol and one wash in $70 \%$ ethanol before they were rinsed in deionized $\mathrm{H}_{2} \mathrm{O}$. For wholemount TUNEL assays, embryos were dehydrated through a graded methanol series $(25 \%, 50 \%, 75 \%$, and twice in $100 \%)$ and stored in $100 \%$ methanol at $-20^{\circ} \mathrm{C}$. After rehydration, sections and wholemount embryos were digested with proteinase $\mathrm{K}(10 \mu \mathrm{g} / \mathrm{mL})$. Embryos were refixed with $4 \%$ paraformaldehyde for 20 minutes at room temperature, followed by several washes in PBS. A detection kit (ApopTag Peroxidase In Situ Apoptosis Detection Kit; Millipore, Billerica, MA) was used to detect levels of apoptotic cell death according to the manufacturer's instructions.

\section{Bioinformatics}

Protein sequences were obtained by querying species-specific databases with human REP1, human REP2, human GDI $\alpha$, yeast REP, and yeast GDI using the BLAST algorithm. ${ }^{22}$ Our analysis was complemented by the more sensitive domain-based methods to identify protein families using Pfam $^{23}$ (GDI domains) and Superfamily ${ }^{24}$ (FAD/NAD binding domains + FAD linked reductase C-terminal domains). Candidate sequences were visually inspected, and REPs and GDIs were distinguished with the use of phylogenetic methods and by the presence of F279 and F135, respectively. ${ }^{25}$ Protein sequences were aligned, and Neighbor Joining Trees were calculated, with 1000 bootstraps, with the use of graphics software (ClustalX 1.80; http://www.clustal.org ${ }^{26}$ with default parameters. Maximum likelihood phylogenetic inference was performed with Phylip ${ }^{27}$ with 100 bootstraps.

\section{Immunoblot Analysis}

Protein extracts were obtained from 20 wild-type and $c h m$ embryos at 6 dpf. Embryos were snap frozen in liquid nitrogen and homogenized in lysis buffer (10 mM Tris, pH 7.5, $10 \mathrm{mM} \mathrm{NaCl}, 1 \%$ SDS, $1 \times$ Protease Inhibitor Cocktail [Roche, Basel Switzerland]) by sonication. Protein concentration was measured with the use of a protein assay kit (BCA Protein Assay kit; Pierce, Rockford, IL). For each sample, $30 \mu \mathrm{g}$ protein was boiled for 5 minutes with an equal volume of SDS sample buffer. Proteins were separated on a $10 \%$ SDS-polyacrylamide gel and transferred to a polyvinylidene difluoride membrane (Immun-Blot; Bio-Rad, Hercules, CA). The membrane was blocked for 2 hours in blocking solution (5\% dry milk, PBS/0.1\% Tween [PBST]), washed three times in PBST, and incubated overnight at $4{ }^{\circ} \mathrm{C}$ with 1:1000 primary anti-REP J905 antibody. ${ }^{14}$ After three washes in PBST, the membrane was incubated with a secondary anti-rabbit IgG horseradish peroxidase conjugate (dilution 1:10,000; GE Healthcare Chalfont St. Giles, Buckinghamshire, UK) for 2 hours. The membrane was washed three times in PBST before chemiluminescence detection using the ECL Western blotting detection kit (GE Healthcare). With the use of a stripping buffer for Western blots (Restore Western Blot Stripping Buffer; Pierce), the membrane was stripped and reprobed with polyclonal anti-gapdh antibody (Abcam, Cambridge, MA) as a loading control for each sample. Image data analyzer software was used to determine the relative abundance of rep protein compared with corresponding levels of control gapdh.

\section{Subcellular Fractionation and In Vitro Prenylation Assays}

Samples were homogenized in hypotonic buffer (50 mM HEPES, pH $7.5,10 \mathrm{mM} \mathrm{NaCl}, 1 \mathrm{mM}$ dithiothreitol, $1 \times$ protease inhibitor cocktail) by sonication. Nuclei were pelleted by centrifugation at $800 \mathrm{~g}$ for 10 minutes before the postnuclear supernatant was collected and centrifuged at $100,000 \mathrm{~g}$ for 1 hour at $4^{\circ} \mathrm{C}$ with a rotor (TLA45; BeckmanCoulter, Fullerton, CA) to obtain cytosolic protein extracts. In vitro prenylation was performed in reaction buffer containing $50 \mathrm{mM}$

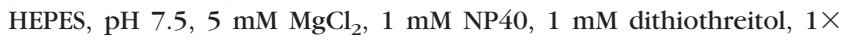
protease inhibitors (Roche), and $1 \times$ phosphatase inhibitors (Sigma). Each $20-\mu \mathrm{L}$ reaction contained $4 \mu \mathrm{M}$ recombinant Rab1a, $100 \mathrm{nM}$ recombinant RGGT, $50 \mathrm{nM}{ }^{3} \mathrm{H}-\mathrm{GGPP}$ supplemented with $5 \mu \mathrm{M}$ unla- 
beled GGPP, and $25 \mu \mathrm{g}$ zebrafish cytosolic extract containing a source of rep. After incubation for 30 minutes at $37^{\circ} \mathrm{C}$, reactions were precipitated by the addition of $10 \% \mathrm{HCl}$ in ethanol and were incubated for 30 minutes at room temperature. As a positive control, zebrafish extracts were used as a source of GGTase-I to prenylate $5 \mu \mathrm{M}$ recombinant human GST-Rac1a using the same reaction conditions but omitting RGGT. Reactions were filtered on glass microfiber filter discs (Whatman, Maidstone, Kent, UK). Each reaction tube was rinsed once with $2 \mathrm{~mL} 100 \%$ ethanol, and each filter was rinsed three times with 2 $\mathrm{mL} 100 \%$ ethanol and counted in a scintillation counter (LS6000SC; Beckman-Coulter). Gel-based assays used $100 \mu \mathrm{g}$ extract $/ 25 \mu \mathrm{L}$ reactions, substituting recombinant Rabs with $2 \mu \mathrm{M}$ recombinant REP and $1 \mu \mathrm{M}$ (instead of $5 \mu \mathrm{M}$ ) unlabeled GGPP. Reactions were incubated for 45 minutes at $37^{\circ} \mathrm{C}$. Loading buffer $(5 \mu \mathrm{L})$ was added to each reaction, and aliquots were boiled for 5 minutes at $95^{\circ} \mathrm{C}$. Aliquots were separated by SDS-PAGE on a $12.5 \%$ gel, and the proteins were fixed by incubation for 1 hour in a solution of $40 \%$ ethanol and $10 \%$ acetic acid. Gels were incubated for 20 minutes in solution (Amplify; Amersham, Piscataway, NJ) before they were dried in a $60^{\circ} \mathrm{C}$ oven and were exposed to autoradiography film for 2 weeks.

\section{Results}

\section{Phenotypes in Homozygous chm Null Zebrafish}

The macroscopic phenotype observed in $\mathrm{chm}^{-/-}$mutants conforms to that previously described by Starr et al. ${ }^{18}$ Among the gross morphologic abnormalities visible from 3 to $4 \mathrm{dpf}$ onward were edema of the heart and abdomen, absence of a functional swim bladder, reduced body length, curvature of the spine, increased dark pigmentation, and microphthalmia (Fig. 1). Mutants also hatched later than their unaffected siblings and retained noticeably larger yolk sacs, possibly because of the lower nutritional requirements of impaired growth. Invariably, $\mathrm{chm}^{-/-}$fish died early, usually by the fifth day (mean survival, $4.8 \mathrm{dpf} ; n=50$ ).

We next examined retinal histology from mutant $\mathrm{chm}^{-/-}$ embryos to characterize the development of retinal disease. Compared with other cell types, photoreceptors occupy a large proportion of the eye from $55 \mathrm{hpf}$, when the formation of outer segments is initiated. ${ }^{28}$ Hence, we hypothesized that photoreceptor cell death would cause reduced eye size as early as $3 \mathrm{dpf}$. At this age, wild-type retinas were laminated, and photoreceptor cells had developed their distinctive morphology (Fig. 1A). Surprisingly, no morphologic evidence of photoreceptor cell death was seen in most sections from $3 \mathrm{dpf}$ or even 4 dpf mutants (Figs. 1A, B). The earliest observed abnormality was a patchy loss of photoreceptors in the outer nuclear layer, with the underlying RPE directly abutting the cells from the inner nuclear layer (Fig. 1C). By $5 \mathrm{dpf}$, multiple cell types throughout the retina were damaged (Figs. 1D, 2). The RPE was hypertrophic and invaded the retina, in some places forming discrete "islands" of pigmented cells; in other areas it was depigmented or absent. Photoreceptors became disorganized and lost their columnar morphology, leaving only regular or pyknotic nuclei in their places, and the outer plexiform layer was almost completely absent. Ordered lamination of amacrine, bipolar, and horizontal cells within the inner nuclear layer was lost, and pyknotic nuclei were present throughout
FIGURE 1. Morphology and retinal histology of chm mutants from $3 \mathrm{dpf}$. Progression of systemic and retinal degeneration from $3 \mathrm{dpf}(\mathbf{A}), 4 \mathrm{dpf}$ (B), $4.5 \mathrm{dpf}(\mathbf{C})$, and $5 \mathrm{dpf}(\mathbf{D})$. Left: comparison of wholemount wildtype (top) and chm (bottom) gross morphology. Middle: coronal retinal sections of wild-type embryos. Right: retinal degeneration of $\mathrm{chm}$ mutant embryos with magnified view of retinal slice. (C, D, red arrows) Area of patchy photoreceptor cell loss in chm retina at $4.5 \mathrm{dpf}$ and $5 \mathrm{dpf}$. (D, white arrow) Area of RPE hypertrophy with invasion of inner nuclear layer. RPE, retinal pigment epithelium; ONL, outer nuclear layer; INL, inner nuclear layer; GCL, ganglion cell layer; ON, optic nerve; p, pyknotic nuclei.

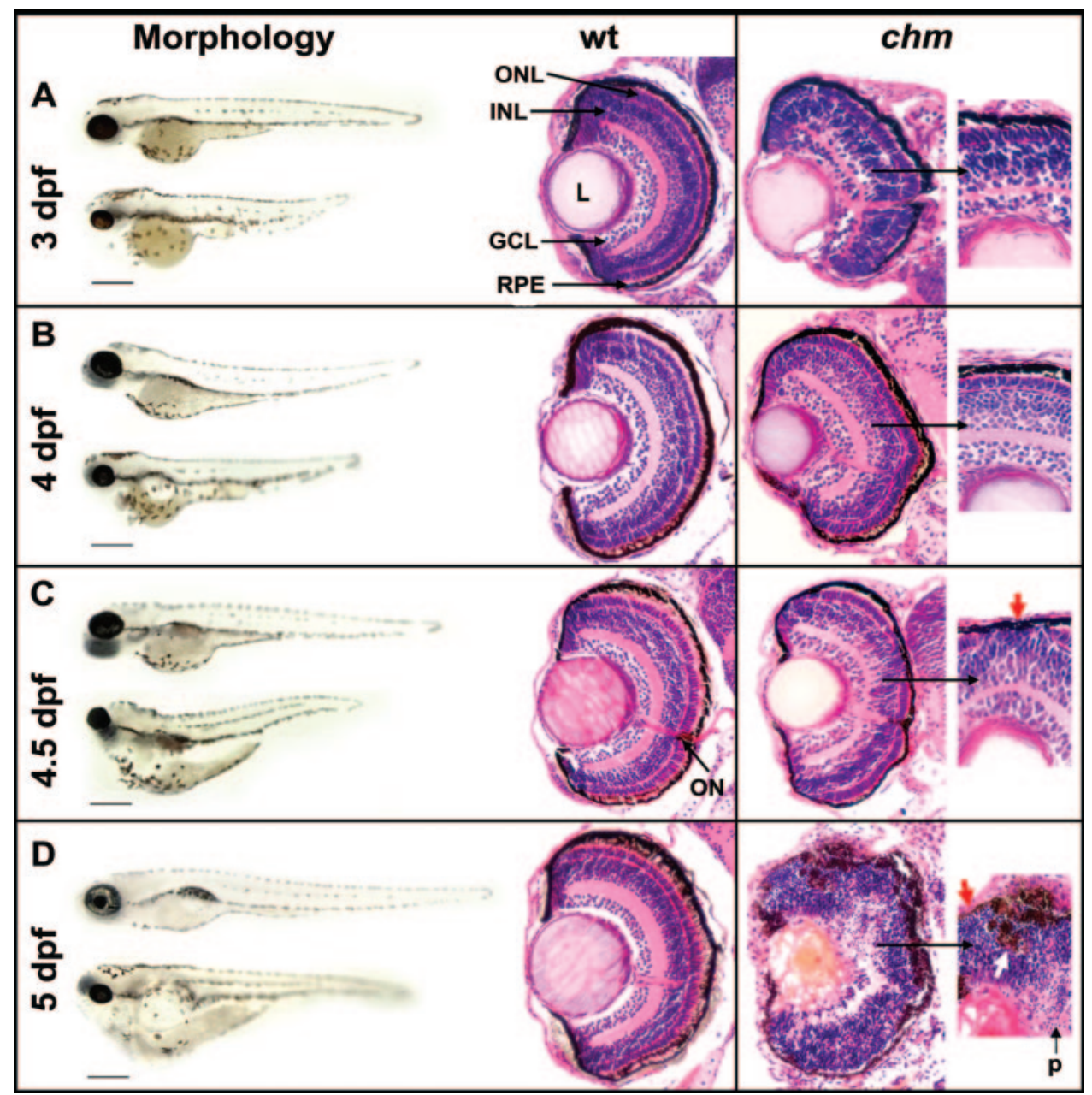




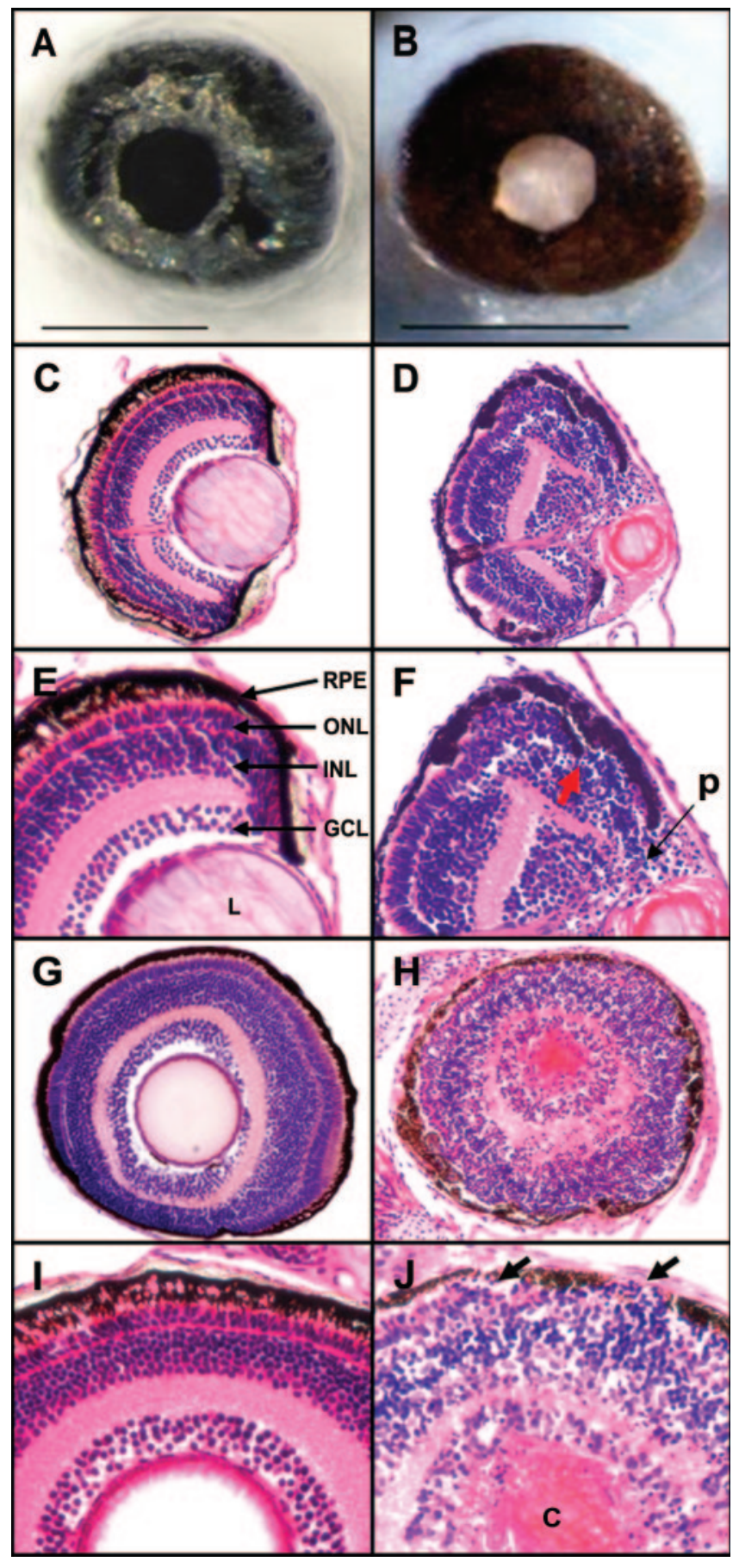

FigURE 2. Retinal histology in $5 \mathrm{dpf} c h m$ mutants. Wild-type (A, C, E, G, I) and chm mutant (B, D, F, H, J) eyes at 5 dpf. Whole eye phenotype showing loss of iridophores in chm mutant (B). (A, B) Scale bars, $250 \mu \mathrm{m}$. (D) Early retinal degeneration in coronal section. (F) Coronal view in detail. (H) Advanced retinal degeneration in parasagittal orientation. (J) Parasagittal view in detail. (F, red arrow) Area of RPE hypertrophy with invasion into the INL and loss of underlying photoreceptor layer. (J, black arrows) Discontinuous RPE. (D, F, H, J) Pyknotic nuclei (p) are present throughout the retina. c, cataractous lens.

the retina, suggesting widespread degeneration (Fig. 2). Severity of the phenotype varied between individuals, suggesting some disparity in the rate of disease progression. For $\mathrm{chm}^{-/-}$ mutants that had a milder phenotype at $5 \mathrm{dpf}(\mathrm{Fig}$. $2 \mathrm{H})$, degeneration appeared to manifest first in the peripheral retina while the central retina remained normal and showed no evidence of cell death. The peripheral RPE invaded the dorsal and ventral retina, and photoreceptors receded from the retinal germinal zone. The outer plexiform layer was discontinuous, and pyknotic nuclei were present in the inner nuclear and ganglion cell layers. In all cases, degeneration of the retina in $\mathrm{chm}^{-/-}$ embryos was surprisingly rapid, usually occurring in 12 hours, between 4.5 and $5 \mathrm{dpf}$. The lens displayed various changes, though there was considerable variation between affected animals. When observed, the lens was often smaller, denser, and impacted and had lens opacities on examination (Figs. 1D, 2).

Wholemount TUNEL staining in $\mathrm{chm}^{-/-}$mutants at $5 \mathrm{dpf}$ revealed widespread cell death across the entire embryo (Fig. 3). Coronal retinal sections showed high levels of apoptosis within the outer nuclear layer involving photoreceptors, with extension into the inner nuclear layer (Fig. 3). Cell death appeared more prolific at the peripheral retina, in accordance

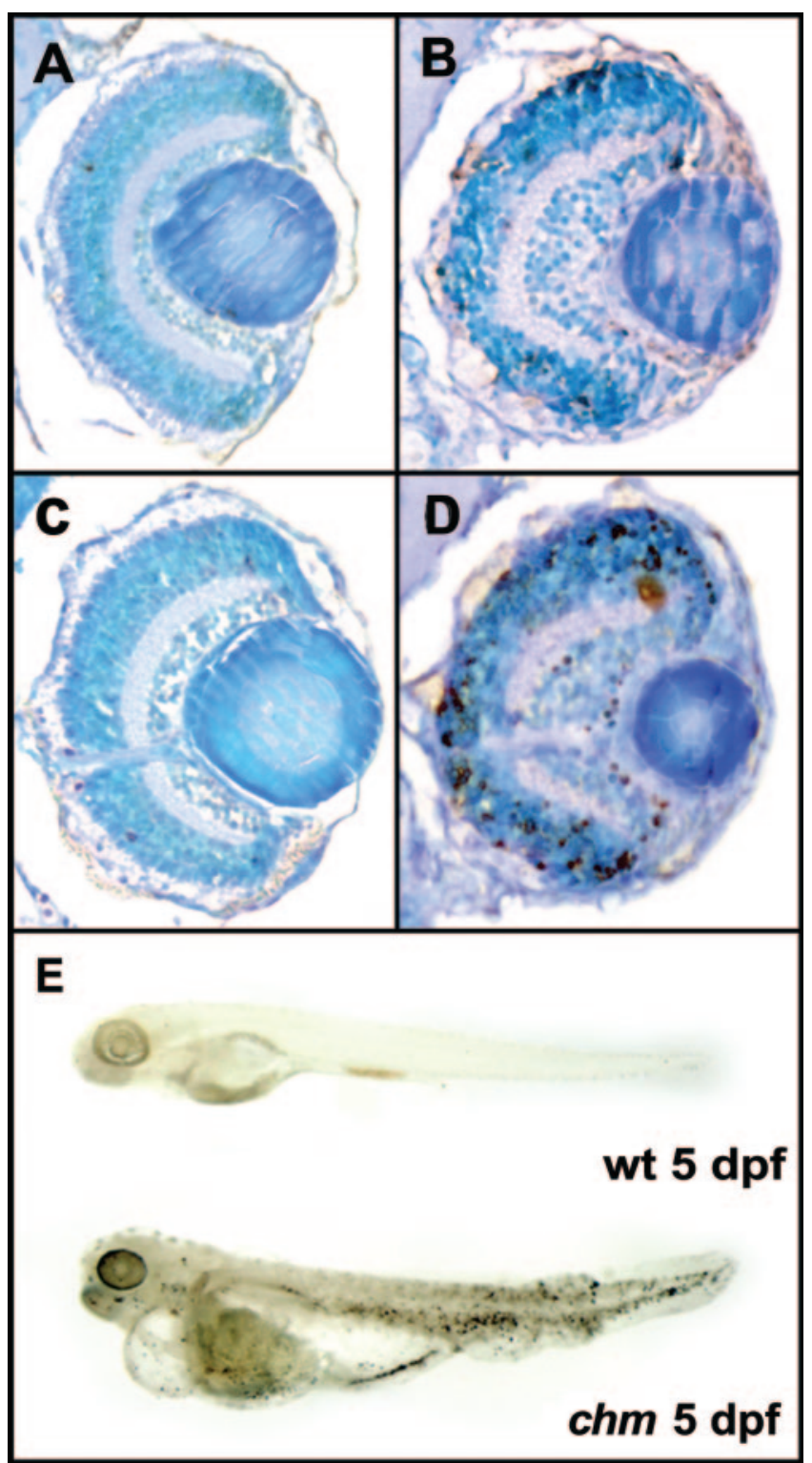

FigURE 3. TUNEL assays on wild-type and $\mathrm{chm}$ mutants. TUNEL assays showing apoptotic cell death in coronal retinal sections of wildtype larvae at (A) $4 \mathrm{dpf}$ and (C) $5 \mathrm{dpf}$, and $\mathrm{chm}$ mutant larvae at (B) 4 dpf and (D) 5 dpf. (E) Wholemount TUNEL assays on wild-type and chm mutants at $5 \mathrm{dpf}$. 
with the histologic findings, and around the exiting optic nerve within the outer nuclear layer. In addition, ganglion cells posterior to the lens capsule were undergoing apoptosis.

\section{Absence of REP Duplication in Fish}

The rapid, degenerative disease observed in $\mathrm{chm}^{-/-}$zebrafish extends beyond the outer retina to cause a more severe retinal, multisystem, and lethal phenotype, more comparable to that seen in yeast with REP mutations (called MRSO) rather than in mice or humans. ${ }^{29}$ Furthermore, we had previously established that in model nonvertebrate organisms, only a single REP gene is present. ${ }^{30}$ We thus hypothesized that zebrafish, unlike mammals, contain a single REP gene whose deletion accounts for the embryonic lethal phenotype.

To test this hypothesis, we decided to reconstruct the evolutionary history of the REP family in vertebrates. We first conducted extensive database searches and identified members of this family in multiple animals. We found two REP isoforms in every eutherian genome (placental mammals; Fig. 4). The closest extant relatives of eutherians are the marsupials and the monotremes. We found a single REP gene in the early release of the platypus genome, a monotreme, and three REP genes in the latest genome release of the marsupial Monodelphis domestica. In amphibia, Xenopus laevis exhibits two isoforms, whereas Xenopus tropicalis exhibits only a single gene. Further down in the vertebrate tree of life, we found a single REP gene in the completed genomes of the zebrafish and the pufferfishes, Takifugu rubripes and Tetraodon nigrovirides (Fig. 4).

Next, we used phylogenetic reconstruction to identify and order gene duplication events and to group the multiple REP isoforms into subfamilies. Mammalian REP1 and REP2 form two distinct groups, suggesting a single origin for each. Human $R E P 2$ is an intronless gene residing in the intron 1 of the gene panopsin. ${ }^{31}$ Our analysis further indicated that all mammalian REP2 genes are intronless.

Opossum REP genes are monophyletic, which indicates two consecutive species-specific duplications. In contrast, the two REP genes in the alloploid frog $X$. laevis are likely accounted by the maintenance of genes from both parent species in a pseudotetraploid genome. ${ }^{32,33}$ All other species, including the three fish genome sequences we analyzed, have a single REP gene. This, together with dating of the origin of REP2 to after the separation of the mammalian lineage, supports our hypothesis that a single REP gene in fish accounts for the gene deletion lethality.

\section{Reduced REP Activity in Choroideremia}

These data established that zebrafish contain a single REP gene, and we know that this is an essential gene in yeast. How can we account for $\mathrm{chm}^{-1-}$ zebrafish survival for approximately 5 days? This would imply that the fish must have some functional REP activity during that period. We hypothesized that a maternal contribution of functional REP activity allows brief survival of $\mathrm{chm}^{-/-}$homozygotes by creating a supply of prenylated rab proteins in the early embryo. In the embryonic zebrafish, all processes occurring before zygotic gene activation rely on maternal products developed during oogenesis and present in the egg at fertilization. These maternal factors must support all cellular functions. ${ }^{34}$ To test this, immunoblot analysis was undertaken on total zebrafish extracts from wild-type and $\mathrm{chm}^{-/-}$mutants with end-stage generalized disease (in this case, at 6 dpf; Fig. 5). Wild-type embryos expressed $87 \%$ rep protein at 6 dpf compared with a loading control, gapdh, whereas $\mathrm{chm}^{-1-}$ mutants exhibited only $15 \%$ of control. This source of rep protein is attributed to residual maternal contribution derived from the yolk sac.

To complement this experiment, we sought to obtain direct evidence for Rep activity in the zebrafish embryos. To measure rep activity in zebrafish, we adapted an in vitro prenylation assay measuring the incorporation of radiolabeled GG diphosphate (GGpp) into rabs. ${ }^{13,14}$ In this assay, whole zebrafish protein extracts were used as a source of rep and were incubated with an in vitro reaction mix containing recombinant RGGT, Rab1a substrate, and ${ }^{3} \mathrm{H}-\mathrm{GG} p \mathrm{p}$. Given the variability between protein extracts, we introduced an internal control in the assay by measuring GGtransferase type I activity, which does not require REP as a cofactor. ${ }^{10}$ When normalized to GGTase-I activity, $5 \mathrm{dpf} \mathrm{chm}^{-/-}$zebrafish extracts exhibited a 2.5 -fold reduction in rep activity compared with wild-type fish extracts (1.88 vs. 0.78 compared with normalized GGTase-I
FigURE 4. Evolutionary history of the REP family. (A) Neighbor Joining tree. (B) Maximum likelihood. Human RabGDI alpha was used as an outgroup. Labels represent bootstrap values (Neighbor Joining, 1000 replicates; maximum likelihood, 100 replicates). Only support levels above $65 \%$ are shown. ci, Ciona intestinalis; ce, Caenorhabditis elegans; dm, Drosophila melanogaster; dr, Danio rerio; gg, Gallus gallus; hs, Homo sapiens; $\mathrm{mm}$, Mus musculus; oa, Ornithorbynchus anatinus; rn, Rattus norvegicus; tn, Tetraodon nigroviridis; tr, Takifugu rubripes; $\mathrm{xl}$, Xenopus laevis; $\mathrm{xt}$, Xenopus tropicalis.

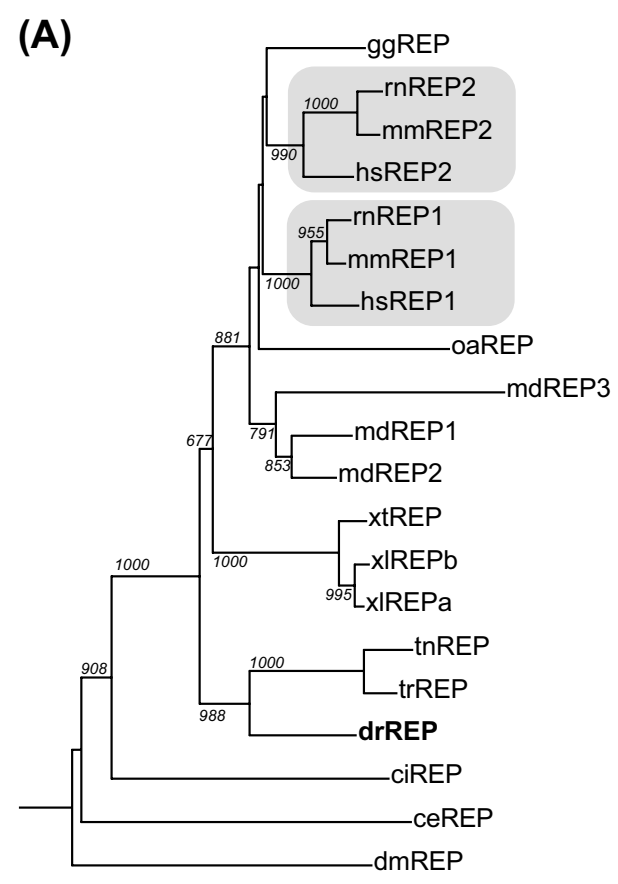

(B)

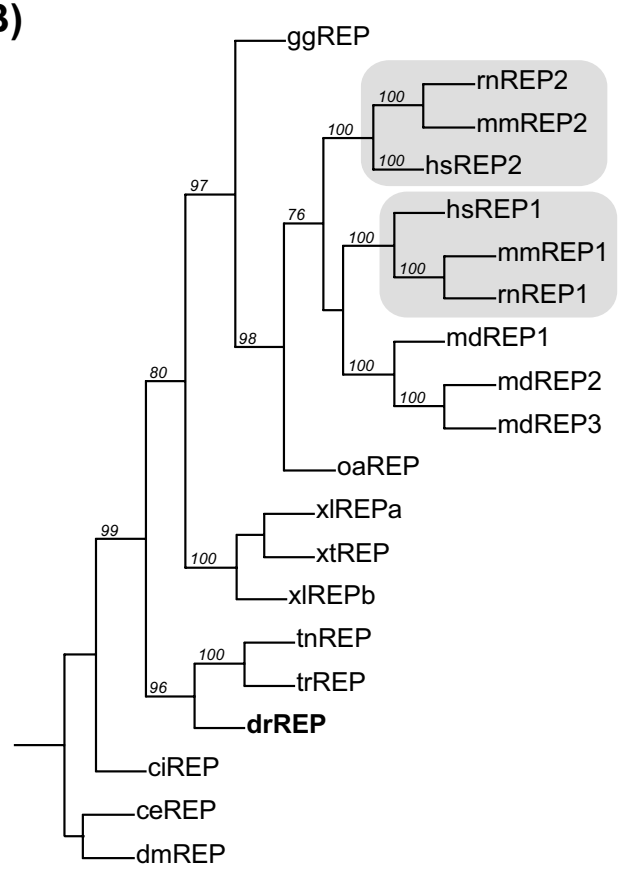


A

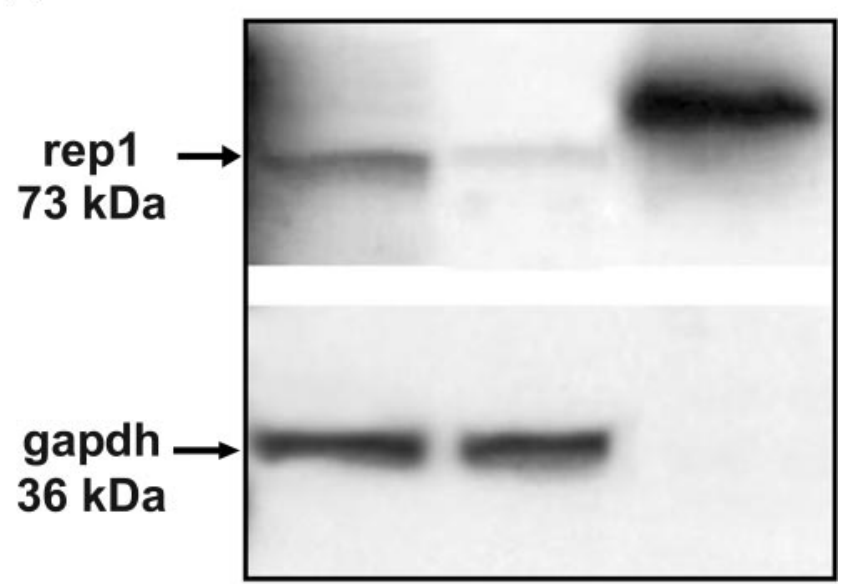

B

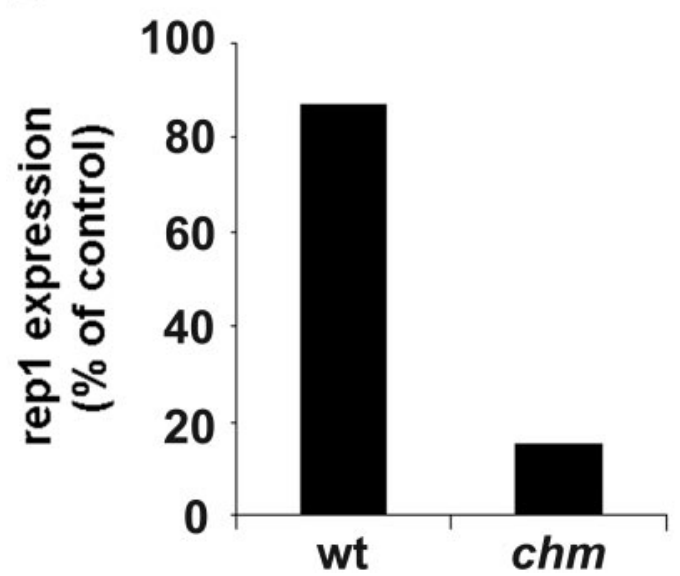

FigURE 5. Immunoblot analysis of the $\mathrm{chm}$ gene product expression (rep). (A) Total zebrafish protein lysates from wild-type and $\mathrm{chm}$ mutant embryos at $6 \mathrm{dpf}$ were subjected to immunoblot analysis. Blot stripped and reprobed with anti-gapdh as a loading control for samples. rREP1, recombinant human REP1 positive control (migrates at a higher molecular weight than zebrafish rep protein). (B) Quantification of rep protein expression relative to gapdh expression with the use of image analyzer software (AIDA imaGenes; Raytest Isotopenmessgeräte $\mathrm{GmbH}$, Straubenhardt, Germany).

activity in the same extract; Fig. 6A). Another prediction from our hypothesis was that the whole-zebrafish cytosolic extracts would have accumulated unprenylated rabs. In this assay, the cytosolic extracts were a source of rab substrates incubated with recombinant REP and RGGT in the presence of ${ }^{3} \mathrm{H}-\mathrm{GG} p \mathrm{p}$. Under these conditions, we observed the presence of ${ }^{3} \mathrm{H}-\mathrm{GG}$ rabs in $\mathrm{chm}^{-/-}$zebrafish extracts but not in wild-type extracts (Fig. 6B). As a positive control, we used a mammalian cell line that accumulates unprenylated Rabs on treatment with the mevalonate pathway inhibitor mevastatin. ${ }^{13}$ This experiment demonstrated the presence of unprenylated rabs in $\mathrm{chm}^{-/-}$ zebrafish, which correlated with the rapid onset of generalized disease. Altogether, our results suggest a mechanism for catastrophic multisystem degeneration in $\mathrm{chm}^{-/-}$zebrafish leading to lethality by 5 to $6 \mathrm{dpf}$.

\section{Discussion}

We present here evidence that the REP family of proteins underwent a mammalian-specific gene duplication event that prevented lethality after loss-of-function mutations in humans. When REP1 is affected, the retinal-restricted phenotype manifests as CHM, whereas any disease resulting from the loss of $R E P 2$ remains unidentified. In $\mathrm{chm}^{-/-}$zebrafish, the loss of the single REP gene is lethal by 5 to $6 \mathrm{dpf}$, and early survival may be attributed to the maternal supply of REP activity.

Human CHM abnormality is limited to the photoreceptors, RPE, and choroid throughout life. In zebrafish, the degenerative process seen in the orthologous gene is more severe. The retina forms relatively normally in the first few days after fertilization; however, after $4.5 \mathrm{dpf}$, we observed rapid degeneration that included all retinal laminae. Because of the speed of the degeneration, it was difficult to elucidate the sequence of cellular loss in the retina. Indeed, one of our initial goals was to determine whether the RPE or photoreceptors were affected first and whether some tissues underwent secondary damage. We quickly realized that the degenerative process was of a general nature and affected most cell types beyond the outer retina and, indeed, the eye. In addition to inner retinal and lens degeneration, $\mathrm{Chm}^{-/-}$zebrafish exhibited uninflated swim bladders, edema of the heart and abdomen, irregular iridophore distribution, and stunted growth that was often associated with spinal curvature.

A

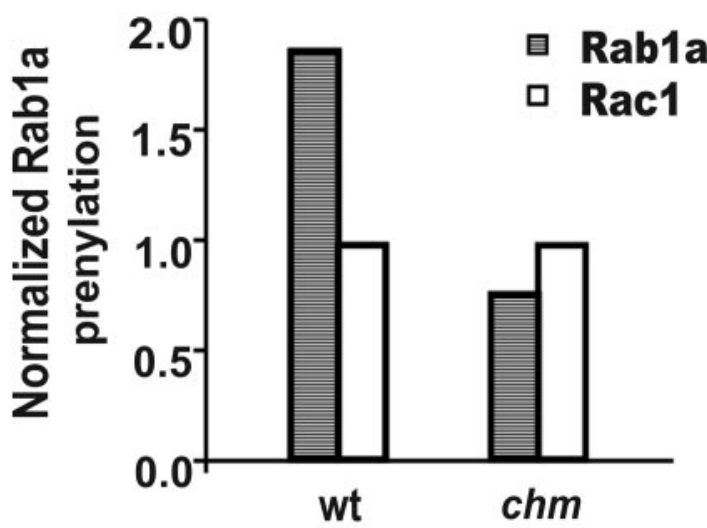

B

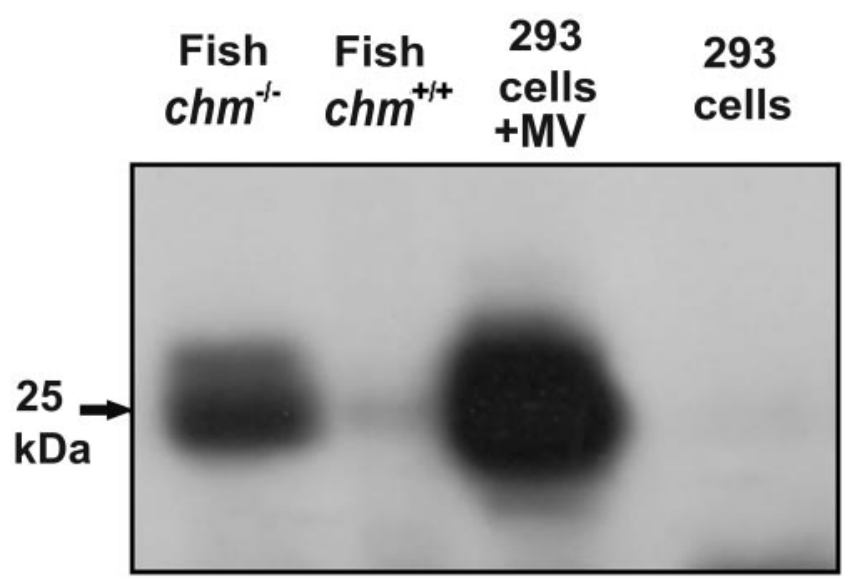

FIGURE 6. REP activity is reduced in $c h m$ mutants leading to accumulation of unprenylated Rabs in the cytosol. (A) Cytosolic extracts from embryonic wild-type (wt) and mutant (chm) zebrafish were used as a source of rep activity and were subjected to in vitro prenylation assays for RGGT and GGT-I. (B) Cytosolic extracts from embryonic wild-type (Fish $\mathrm{chm}^{+/+}$) and mutant (Fish $\mathrm{chm}^{-/-}$) zebrafish, HEK293 cells with $(293$ cells + MV), or without ( 293 cells) mevastatin were subjected to gel-based in vitro prenylation assays with radiolabeled GGpp. 
While this work was in progress, Krock et al. ${ }^{19}$ reported on the retinal phenotype of $\mathrm{chm}^{-/-}$fish and showed evidence of RPE and photoreceptor degeneration. Our observations concur with the reported observations, including normal retinal lamination, and various obvious pathologic changes in the RPE and photoreceptors. However, that work was limited by the absence of time-course studies given that the reported analysis was restricted to $4.5 \mathrm{dpf}$ embryos. This precedes the onset of extensive degeneration of the inner retina (Figs. 1, 2). A mere 12 hours later, the retina looks very different, and generalized degeneration is evident in all cell layers. Therefore, one important issue is whether $\mathrm{chm}^{-/-}$fish retinal degeneration bears any resemblance to human CHM (in other words, whether $\mathrm{chm}^{-/-}$fish may be used as a faithful model of CHM). Our results indicate that it does not.

The extreme abnormalities of zebrafish $\mathrm{chm}^{-/-}$can be explained if this disorder is considered to be a null mutation in an essential gene rather than strictly a retinal degeneration. Embryonic essential genes in zebrafish are classified as those that, when disrupted, result in a phenotype visible by $5 \mathrm{dpf}$ that is invariably lethal. ${ }^{35}$ By this definition, $c h m$ is an embryonic lethal gene because REP is essential for embryonic development. Our hypothesis to explain the generalized degenerative process followed by death before $6 \mathrm{dpf}$ in $\mathrm{chm}^{-/-}$ zebrafish is as follows. The maternal supply of rep allows for enough REP activity to ensure the normal development of the early embryo. Maternal REP activity allows the prenylation of the rab GTPases expressed by these cells. A significant maternally derived complement of mRNA or rep protein must be highly stable to persist for several days in zebrafish $\mathrm{chm}^{-/-}$ mutants. ${ }^{36-39}$ Indeed, maternal products have been shown to function beyond the midblastula transition, when most maternal mRNAs are degraded. ${ }^{34,40}$ In this scenario, another variable that could explain variation in disease severity between individuals is the initial dosage of maternal rep received by the embryo. ${ }^{38}$ As the finite supply of maternal mRNA for rep declines, so does the expression of rep protein. Nevertheless, experiments in yeast have shown that reduced levels of REP prenylation activity are sufficient to maintain a functional Rab pool in vivo, and REP function is only transiently required to support normal growth. ${ }^{41}$ An additional specificity of REP function is that it serves to activate Rabs, and Rab activity appears to be the required function for cell viability. In yeast, prenylated Rabs are relatively stable, requiring at least a single generation for turnover, and only $10 \%$ to $20 \%$ of normal levels of prenylated (membrane-bound) Rabs are sufficient to allow cellular growth. ${ }^{41}$ Therefore, declining REP activity would manifest itself phenotypically only after the pool of prenylated Rabs had turned over. At that point, Rab dysfunction would reach a level inconsistent with the maintenance of normal membrane trafficking processes, and generalized degeneration would ensue.

Our analysis revealed that all mammalian REP 2 genes are intronless, further supporting a single gene duplication event through reverse transcription. These data strongly suggests that REP1, rather than REP2, is the ancestral form, as proposed previously. ${ }^{42}$ Many autosomal genes that arose by retroposition from X-linked genes developed testis expression, presumably to avoid $\mathrm{X}$ inactivation during spermatogenesis, when the retrogene may contribute complementary function(s) to the parental paralogue. ${ }^{43-45}$ It would be interesting to test whether REP2 follows the same male-specific bias to determine what specific function it may perform during spermatogenesis and whether REP2 malfunction may be associated with infertility or other germline defects in mammals.

It appears then that the absence of a second compensatory REP limits the application of zebrafish as a model for choroideremia. REP2 plays an essential role in disease pathogenesis in humans. Hence, the introduction of a transgenic copy of human REP2 gene into $\mathrm{chm}^{-/-}$zebrafish may create a model for the study of human CHM disease. Conversely, a model organism with a single REP will be of use in characterizing therapies that aim to boost REP1 activity, such as novel drug classes that allow read-through of nonsense mutations. ${ }^{46-48}$ Such approaches will afford the zebrafish a continued role in efforts to further understand and seek treatments for CHM.

\section{Acknowledgments}

The authors thank Lorraine Lawrence for technical assistance with histologic specimens and Neil Vargesson for setup of the zebrafish facility and initial breeding of the chm strain.

\section{References}

1. Scriver CR, Sly WS, Beaudet AL, Valle D, eds. The Metabolic and Molecular Bases of Inberited Disease. New York: McGraw-Hill, Inc; 1995;4311-4324.

2. Heckenlively JR, Bird AJ. Choroideremia. In: Heckenlively JR, Daiger SP, eds. Retinitis Pigmentosa. Philadelphia: JB Lippincott; 1988; 176-187.

3. Cremers FP, van de Pol DJ, van Kerkhoff LP, Wieringa B, Ropers $\mathrm{HH}$. Cloning of a gene that is rearranged in patients with choroideraemia. Nature. 1990;347:674-677.

4. Seabra MC, Brown MS, Goldstein JL. Retinal degeneration in choroideremia: deficiency of rab geranylgeranyl transferase. Science. 1993;259:377-381.

5. Seabra MC, Brown MS, Slaughter CA, Sudhof TC, Goldstein JL. Purification of component A of Rab geranylgeranyl transferase: possible identity with the choroideremia gene product. Cell. 1992; 70:1049-1057.

6. Cremers FP, Molloy CM, van de Pol DJ, et al. An autosomal homologue of the choroideremia gene colocalizes with the Usher syndrome type II locus on the distal part of chromosome 1q. Hum Mol Genet. 1992;1:71-75.

7. Andres DA, Seabra MC, Brown MS, et al. cDNA cloning of component A of Rab geranylgeranyl transferase and demonstration of its role as a Rab escort protein. Cell. 1993;73:1091-1099.

8. Seabra MC, Wasmeier C. Controlling the location and activation of Rab GTPases. Curr Opin Cell Biol. 2004;16:451- 457.

9. Zerial M, McBride H. Rab proteins as membrane organizers. Nat Rev Mol Cell Biol. 2001;2:107-117.

10. Casey PJ, Seabra MC. Protein prenyltransferases. J Biol Chem. 1996;271:5289-5292.

11. Desnoyers L, Anant JS, Seabra MC. Geranylgeranylation of Rab proteins. Biochem Soc Trans. 1996;24:699-703.

12. Seabra MC. New insights into the pathogenesis of choroideremia: a tale of two REPs. Opbthalmic Genet. 1996;17:43-46.

13. Seabra MC, Ho YK, Anant JS. Deficient geranylgeranylation of Ram/Rab27 in choroideremia. J Biol Chem. 1995;270:2442024427.

14. Tolmachova T, Anders R, Abrink M, et al. Independent degeneration of photoreceptors and retinal pigment epithelium in conditional knockout mouse models of choroideremia. J Clin Invest. 2006;116:386-394.

15. van den Hurk JA, Hendriks W, van de Pol DJ, et al. Mouse choroideremia gene mutation causes photoreceptor cell degeneration and is not transmitted through the female germline. Hum $\mathrm{Mol}$ Genet. 1997;6:851-858.

16. Shi W, van den Hurk JA, Alamo-Bethencourt V, et al. Choroideremia gene product affects trophoblast development and vascularization in mouse extra-embryonic tissues. Dev Biol. 2004;272:5365.

17. Goldsmith P, Harris WA. The zebrafish as a tool for understanding the biology of visual disorders. Semin Cell Dev Biol. 2003;14:1118.

18. Starr CJ, Kappler JA, Chan DK, Kollmar R, Hudspeth AJ. Mutation of the zebrafish choroideremia gene encoding Rab escort protein 1 devastates hair cells. Proc Natl Acad Sci US A. 2004;101:25722577. 
19. Krock BL, Bilotta J, Perkins BD. Noncell-autonomous photoreceptor degeneration in a zebrafish model of choroideremia. Proc Natl Acad Sci U S A. 2007;104:4600-4605.

20. Westerfield M. The Zebrafish Book: A Guide for the Laboratory Use of Zebrafish (Danio rerio). 4th ed. Seattle: University of Oregon Press; 2000.

21. Kimmel CB, Ballard WW, Kimmel SR, Ullmann B, Schilling TF. Stages of embryonic development of the zebrafish. Dev Dyn. 1995;203:253-310.

22. Altschul SF, Gish W, Miller W, Myers EW, Lipman DJ. Basic local alignment search tool. J Mol Biol. 1990;215:403-410.

23. Bateman A, Coin L, Durbin R, et al. The Pfam protein families database. Nucleic Acids Res. 2004;32:D138-141.

24. Wilson D, Madera M, Vogel C, Chothia C, Gough J. The SUPERFAMILY database in 2007: families and functions. Nucleic Acids Res. 2007;35:D308-D313.

25. Pylypenko O, Rak A, Reents R, et al. Structure of Rab escort protein-1 in complex with Rab geranylgeranyltransferase. Mol Cell. 2003; 11:483-494.

26. Thompson JD, Gibson TJ, Plewniak F, Jeanmougin F, Higgins DG The CLUSTAL_X windows interface: flexible strategies for multiple sequence alignment aided by quality analysis tools. Nucleic Acids Res. 1997;25:4876-4882

27. Felsenstein J. PHYLIP-Phylogeny Inference Package (version 3.2). Cladistics. 1989;5:164-166

28. Schmitt EA, Dowling JE. Early retinal development in the zebrafish, Danio rerio: light and electron microscopic analyses. J Comp Neurol. 1999;404:515-536.

29. Waldherr M, Ragnini A, Schweyer RJ, Boguski MS. MRS6-yeast homologue of the choroideraemia gene. Nat Genet. 1993;3:193194.

30. Rasteiro R, Pereira-Leal JB. Multiple domain insertions and losses in the evolution of the Rab prenylation complex. BMC Evol Biol. 2007;7:140.

31. Halford S, Freedman MS, Bellingham J, et al. Characterization of a novel human opsin gene with wide tissue expression and identification of embedded and flanking genes on chromosome $1 \mathrm{q} 43$. Genomics. 2001;72:203-208.

32. Evans BJ. Ancestry influences the fate of duplicated genes millions of years after polyploidization of clawed frogs (Xenopus). Genetics. 2007;176-1119-1130.

33. Evans BJ, Kelley DB, Tinsley RC, Melnick DJ, Cannatella DC. A mitochondrial DNA phylogeny of African clawed frogs: phylogeog- raphy and implications for polyploid evolution. Mol Phylogenet Evol. 2004;33:197-213.

34. Pelegri F. Maternal factors in zebrafish development. Dev Dyn. 2003;228:535-554.

35. Amsterdam A, Nissen RM, Sun Z, Swindell EC, Farrington S, Hopkins N. Identification of 315 genes essential for early zebrafish development. Proc Natl Acad Sci U S A. 2004;101:12792-12797.

36. Driever W, Solnica-Krezel L, Schier AF, et al. A genetic screen for mutations affecting embryogenesis in zebrafish. Development. 1996;123:37- 46 .

37. Golling G, Amsterdam A, Sun Z, et al. Insertional mutagenesis in zebrafish rapidly identifies genes essential for early vertebrate development. Nat Genet. 2002;31:135-140.

38. Gross JM, Perkins BD, Amsterdam A, et al. Identification of zebrafish insertional mutants with defects in visual system development and function. Genetics. 2005;170:245-261.

39. Kane DA, Maischein HM, Brand M, et al. The zebrafish early arrest mutants. Development. 1996;123:57-66.

40. Wagner DS, Dosch R, Mintzer KA, Wiemelt AP, Mullins MC. Maternal control of development at the midblastula transition and beyond: mutants from the zebrafish II. Dev Cell. 2004;6:781-790.

41. Alory C, Balch WE. Molecular basis for Rab prenylation. J Cell Biol. 2000; 150:89-103.

42. Alory C, Balch WE. Molecular evolution of the Rab-escort-protein/ guanine-nucleotide-dissociation-inhibitor superfamily. $\mathrm{Mol} \mathrm{Biol}$ Cell. 2003;14:3857-3867.

43. Betran E, Emerson JJ, Kaessmann H, Long M. Sex chromosomes and male functions: where do new genes go? Cell Cycle. 2004;3: 873-875.

44. Emerson JJ, Kaessmann H, Betran E, Long M. Extensive gene traffic on the mammalian X chromosome. Science. 2004;303:537-540.

45. Vinckenbosch N, Dupanloup I, Kaessmann H. Evolutionary fate of retroposed gene copies in the human genome. Proc Natl Acad Sci US A. 2006;103:3220-3225.

46. Mankin AS, Liebman SW. Baby, don't stop! Nat Genet. 1999;23:810 .

47. Welch EM, Barton ER, Zhuo J, et al. PTC124 targets genetic disorders caused by nonsense mutations. Nature. 2007;447:87-91.

48. Moosajee M, Gregory-Evans K, Ellis CD, Seabra MC, Gregory-Evans CY. Translational bypass of nonsense mutations in zebrafish rep1, pax2.1 and lamb1 highlights a viable therapeutic option for untreatable genetic eye disease. Hum Mol Genet. 2008;17:39874000 . 